\title{
Investigation of Gremlin 1, COL15A1 immunoreactivity and the relationship between microvessel density and Gremlin 1 in papillary renal cell carcinoma and chromophobe renal cell carcinoma
}

\author{
Sevgul Kara*, Metin Karakok
}

\begin{abstract}
Objective: The current study aimed to investigate expressions of Gremlin 1 (GREM1) (a bone morphogenetic protein antagonist and a proangiogenic factor) and COL15A1 (Collagen type XV alpha-1, encodes the proteoglycans located in various human tissues and particularly in the basement membrane) immunohistochemically in papillary renal cell carcinoma (PRCC) and chromophobe renal cell carcinoma (CRCC) in order to assess the relationship between these markers and said tumors and also explore associations of GREM1 with angiogenesis, tumor necrosis and tumor diameter by looking at the microvascular density (MVD) through the expression of COL15A1 in vascular endothelium.
\end{abstract}

Material and Method: GREM1 and COL15A1 expressions were investigated in 20 PRCC and 39 CRCC patients. Cytoplasmic staining with GREM1 and COL15A1 was examined. Microvascular structures stained with COL15A1 were examined in order to evaluate angiogenic profile.

Results: In CRCC, GREM1 staining was statistically significant in tumor tissues compared to intact tissues ( $p=0.006$ ). The relationship between MVD and GREM1 staining was statistically significant in PRCC ( $\mathrm{p}=0.007)$. Cytoplasmic staining with COL15A1 observed in PRCC was statistically significant $(\mathrm{p}=0.005)$.

Conclusion: Positive GREM1 staining observed in both tumor groups and much higher expression of this marker particularly in the tubular epithelium of the neighboring normal tissue supports our argument that this gene might be a tumor suppressor gene.

Keywords: Chromophobe renal cell carcinoma, papillary renal cell carcinoma, Gremlin 1, collagen type XV alpha-1, angiogenesis

\section{Introduction}

Gremlin 1 (GREM1) is a bone morphogenetic protein (BMP) antagonist and a novel proangiogenic factor (1). GREM1 suppresses transforming growth factor-beta (TGF- $\beta$ ) signaling by binding to BMP-2, BMP-4 and BMP-7 and blocking the interaction of these ligands with their receptors. BMP family is largest subfamily of TGF- $\beta$ superfamily of growth factors which are involved in several functions including angiogenesis, proliferation, apoptosis, differentiation, chemotaxis and production of extracellular matrix in many cells (2). BMP and BMP antagonists like GREM1 have been demonstrated in the pathogenesis of nephropathy and regulation of kidney development. GREM1 directly interacts with cancer cells in a BMP-independent manner and modulates angiogenesis. Although the role of GREM1 in the pathogenesis and underlying mechanism of renal (kidney) cancer has been shown through GREM1 gene expression, its other actions have not been clearly elucidated (1).

Epigenetic mechanisms may play a key role in the regulation of GREM1 expression. Recent studies have indicated that methylation of GREM1 promoter region is strongly associated with the development of certain types of cancer (1).

Collagen type XV alpha-1 (COL15A1) is a large proteoglycan that has been demonstrated in all human tissues. By light microscopy, it was localized to most epithelial and all nerve, muscle and endothelial basement membrane zones except for the glomerular capillaries or hepatic/splenic sinusoids (3).

COL15A1 gene encodes the proteoglycans located in various human tissues and particularly in the basement membrane and 
it is believed to have a major role in signaling pathways (3). Hypermethylation of the promoter region of the COL15A1 gene was reported in patients with clear cell renal cell carcinoma (CCRCC) (4) but COL15A1 gene has not been previously studied in patients with papillary renal cell carcinoma (PRCC) and chromophobe renal cell carcinoma (CRCC).

Kidney cancer is the thirteenth most common cancer worldwide and tenth most common cancer in Europe. According to the World Health Organization data, 271,000 new cases were identified globally in 2008. Although the incidence of kidney cancer stabilized in recent years and even declined in some European countries, metastasis occurs in $20 \%$ to $30 \%$ of diagnosed patients. Kidney cancers remain life-threatening since patients with kidney cancers experience relapse and metastasis following nephrectomy. Genetic and molecular studies contribute to the understanding of tumor pathogenesis and may also be used for determination of the tumor type. Additionally, such studies would serve as a guide for developing individualized and targeted therapies. Renal cell carcinoma (RCC) is the most common malignant kidney tumor and has 3 major subtypes including CCRCC which has the highest incidence and PRCC and CRCC. These subtypes differ in their histological, morphological and genetic characteristics ally as well as clinical course (5).

In one study, it was concluded that hypermethylation of the promoter region of GREM1 gene as shown in patients with CCRCC may have an impact on the development of this type of cancer and angiogenesis (1). Another study has also demonstrated deoxyribonucleic acid (DNA) hypermethylation in PRCC (6). However, no study was identified in literature on GREM1 in PRCC and CRCC. Hypermethylation of the promoter region of the COL15A1 gene was reported in patients with CCRCC (4) but COL15A1 gene has not been previously studied in patients with PRCC and CRCC.

In the present study, we aimed to investigate GREM1 and COL15A1 expressions in PRCC and CRCC immunohistochemically in order to assess the relationship between these markers and studied tumors and explore the association of GREM1 with angiogenesis, age, tumor necrosis and tumor diameter by looking at the microvascular density (MVD) through the expression of COL15A1 in the vascular endothelium.

\section{Material and Methods}

Digital archives of Gaziantep University Faculty of Medicine, Department of Pathology were screened and 59 cases of neprectomy were identified in patients diagnosed with CRCC and PRCC between 2002 and 2011. Paraffin-embedded blocks were retrieved from the block archive in order to prepare 4 micron thick sections of tissue (one from tumor tissue and one from normal kidney parenchyma) for each patient and GREM1 and COL15A1 antibodies were studied using an immunohistochemical method.

Gremlin 1 (GREM1 polyclonal antibody PAB 14845, Abnova Corp.) and COL15A1 (polyclonal antibody NBP1-91087, Novus Biologicals) antibodies were studied with Leica Bond Max (Leica Biosystems) using a Bond Polymer Refine Detection kit. Preparations obtained were examined by 2 pathologists under light microscope. Normal tissue staining pattern was compared with tumor tissue staining pattern.

\section{Gremlin 1 staining and its interpretation}

Areas of cytoplasmic staining with GREM1 were examined both in the tumor tissue and normal kidney tissue of each patient under light microscope. Cytoplasmic staining observed in the renal tubular epithelium of the normal tissue was considered positive. Based on the pattern of staining, no staining was assigned a score of 0 , weak and diffuse staining was assigned score 1 positive and diffuse and strong staining was assigned score 2 positive. For convenience in statistical analysis, score 1 and 2 were combined in a single parameter to indicate "staining present (positive)" and score 0 indicated "staining absent (negative)".

\section{Collagen type XV alpha-1 staining and its interpretation}

Areas of cytoplasmic staining with COL15A1 in tumor cells were examined in tumor tissues. Based on the pattern of staining, no staining was assigned a score of 0 , weak and diffuse staining was assigned score 1 positive and diffuse and strong staining was assigned score 2 positive. For convenience in statistical analysis, score 1 and 2 were combined in a single parameter to indicate "staining present (positive)" and score 0 indicated "staining absent (negative)".

\section{Microvascular density}

Considering that COL15A1 also serves as an endothelial marker, microvessels stained with COL15A1 were counted under light microscopy at a magnification of $\mathrm{x} 400$ in 5 fields with a clear view of the lumen in randomly selected tumor tissues and normal tissues (7).

\section{Statistical analysis}

For statistical analyses, Fischer exact test and Mann-Whitney $\mathrm{U}$ test were used to conduct appropriate comparisons between tumor groups. SPSS for Windows software was used for statistical analyses. For comparisons, a $p$ value less than 0.05 was considered statistically significant.

\section{General findings}

RCC cases included $20 \mathrm{PRCC}$ and $39 \mathrm{CRCC}$ cases. The mean age was 56.5 years in PRCC patients and 54.15 years in CRCC 
patients. The mean tumor size was $6.34 \mathrm{~cm}$ for PRCC and 8.24 for CRCC.

\section{Immunohistochemical findings}

GREM1 and COL15A1 markers were studied in 59 RCC patients using immunohistochemical methodology. Associations of these markers with the type and diameter of tumor, age, sex, MVD and tumor necrosis were evaluated.

Cytoplasmic staining of tumor tissues with GREM1 was assessed in the two tumor groups separately (Figure 1). Staining was negative in $80 \%$ (score 0 ) and positive in $20 \%$ of PRCC cases (Images 1, 2).

Among CRCC cases, staining was absent in $20.5 \%$ and positive staining was found in $69.2 \%$ (score 1) and $10.3 \%$ (score 2) (Table 1 and Images 3, 4).

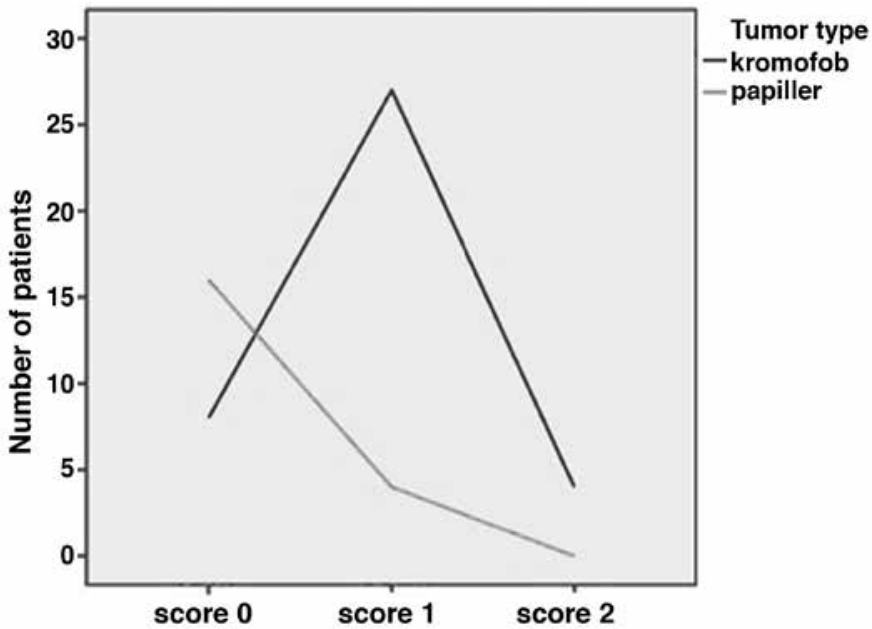

Figure 1. The degree of Gremlin 1 staining in tumor tissues as shown by the number of chromophobe renal cell carcinoma and papillary renal cell carcinoma patients

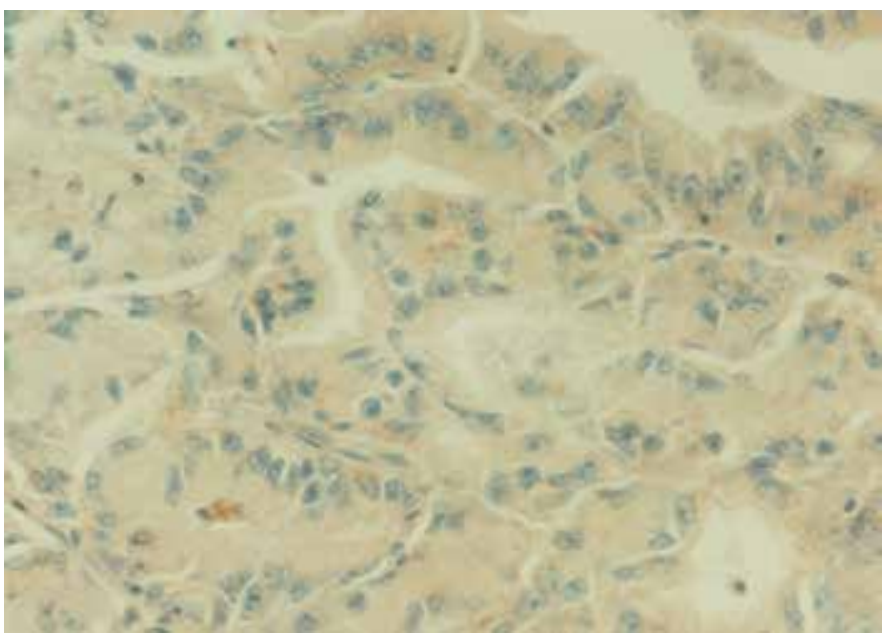

Image 1. Gremlin 1 expression in papillary renal cell carcinoma (score 0), x400
When cytoplasmic GREM1 staining of the tubular epithelial cytoplasm was evaluated separately in the healthy kidney tissues of the two tumor groups, no staining was detected in $35 \%$ of PRCC patients and positive staining was found in $60 \%$ (score 1) and 5\% (score 2). For CRCC patients, staining was absent in $7.7 \%$ and positive staining was observed in $79.5 \%$ (score 1) and $12.8 \%$ (score 2) (Image 5, Table 2).

In PRCC, cytoplasmic GREM1 staining was statistically nonsignificant between tumor tissue versus normal tissue $(\mathrm{p}=0.249)$.

In CRCC, cytoplasmic positive staining with GREM1 was statistically significant for tumor tissue in comparison to normal tissue $(\mathrm{p}=0.006)$.

Analysis of the relationship between GREM1 expression and sex did not reveal a statistically significant difference between

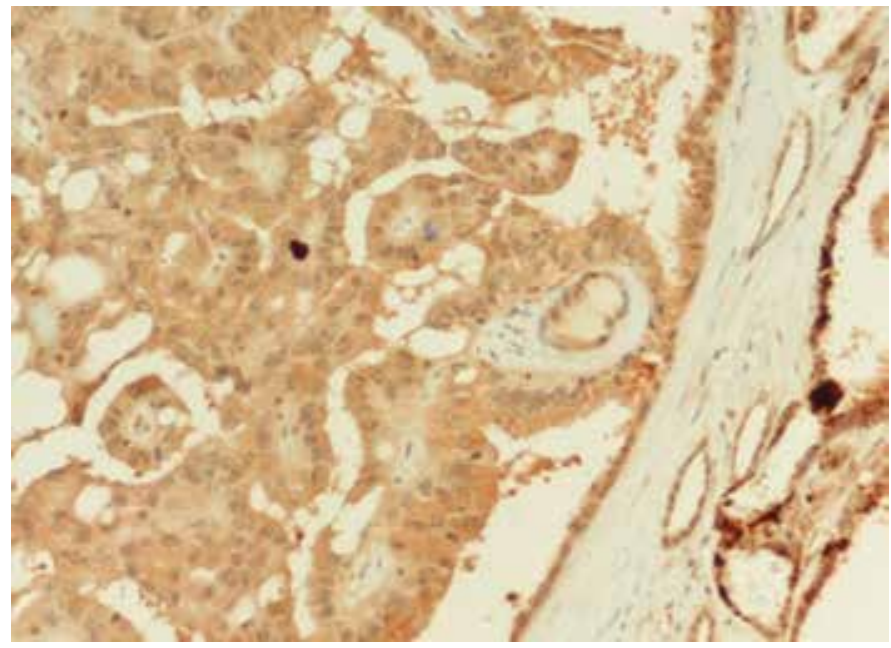

Image 2. Cytoplasmic expression of Gremlin 1 in papillary renal cell carcinoma (score 1) x200

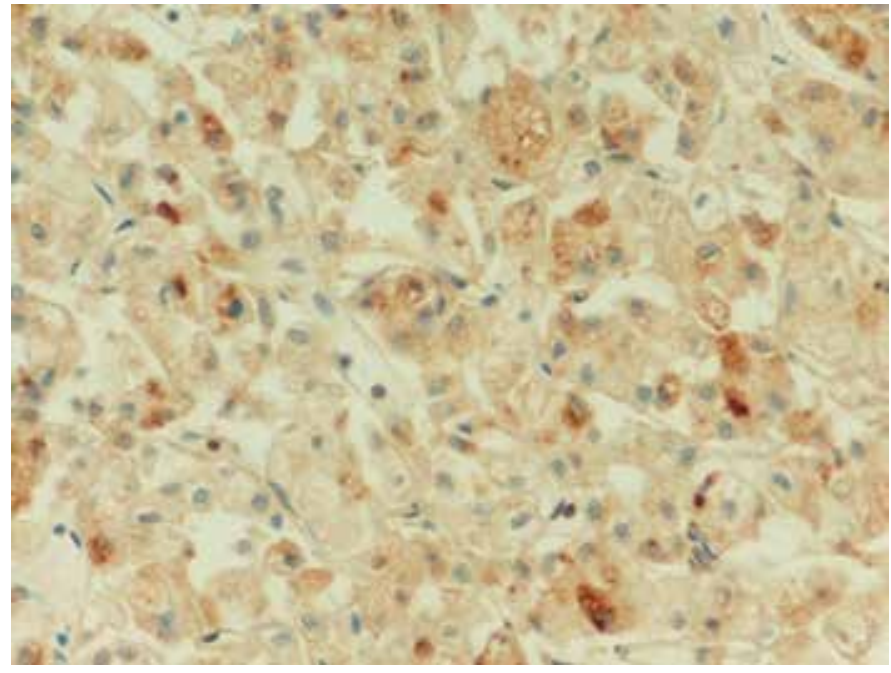

Image 3. Cytoplasmic expression of Gremlin 1 in chromophobe renal cell carcinoma (score 1) x400 
males $(n=6)$ and females $(n=13)$ among CRCC patients.

GREM1 expression did not differ statistically significantly

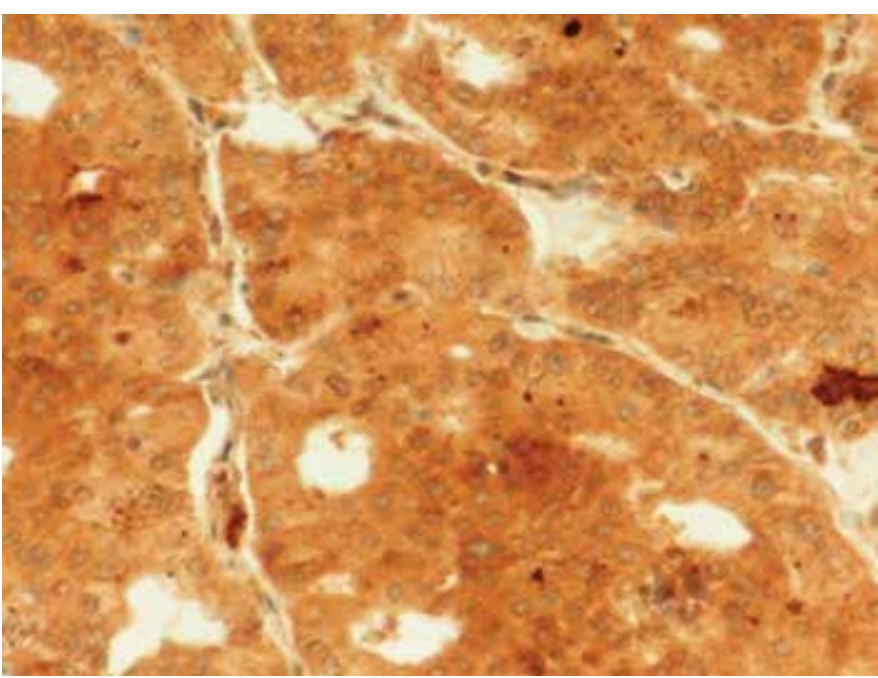

Image 4. Cytoplasmic expression of Gremlin 1 in chromophobe renal cell carcinoma (score 2) $\times 400$

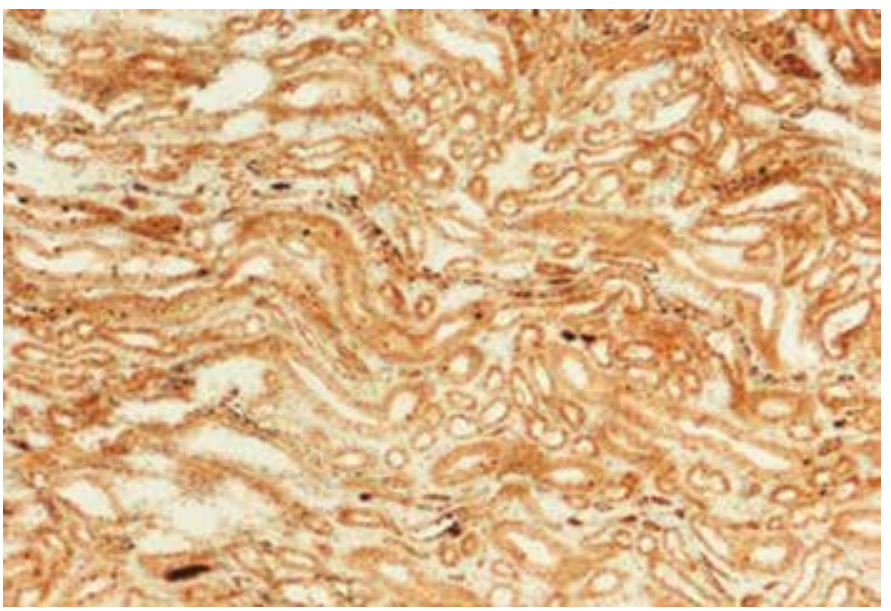

Image 5. Gremlin 1 expression in the renal tubules of healthy kidney tissue in a chromophobe renal cell carcinoma patient (score 2) $\times 100$ with respect to necrosis in CRCC patients (necrosis present: 13 patients, necrosis absent: 13 patients). Tumor diameter did not show a statistically significant association with GREM1 expression among CRCC patients (Table 3).

Evaluation of the cytoplasmic staining with COL15A1 in the tumor tissues of two tumor groups separately showed negative staining in $70 \%$ of PRCC patients and score 1 positive staining in $30 \%$ of patients (Images 6,7 ). Negative staining with COL15A1 was observed in $97.4 \%$ of CRCC patients and positive staining in $2.6 \%$ (Image 8). Higher rate of staining observed in PRCC was statistically significant $(\mathrm{p}=0.0059)$ (Table 4$)$.

No significant association was found between COL15A1 expression and age, sex, presence of tumor necrosis or tumor diameter in PRCC patients (Table 5).

Analysis of the association of tumor COL15A1 expression and sex did not show a statistically significant difference between males $(n=15)$ and females $(n=5)$ among PRCC patients. Tumor

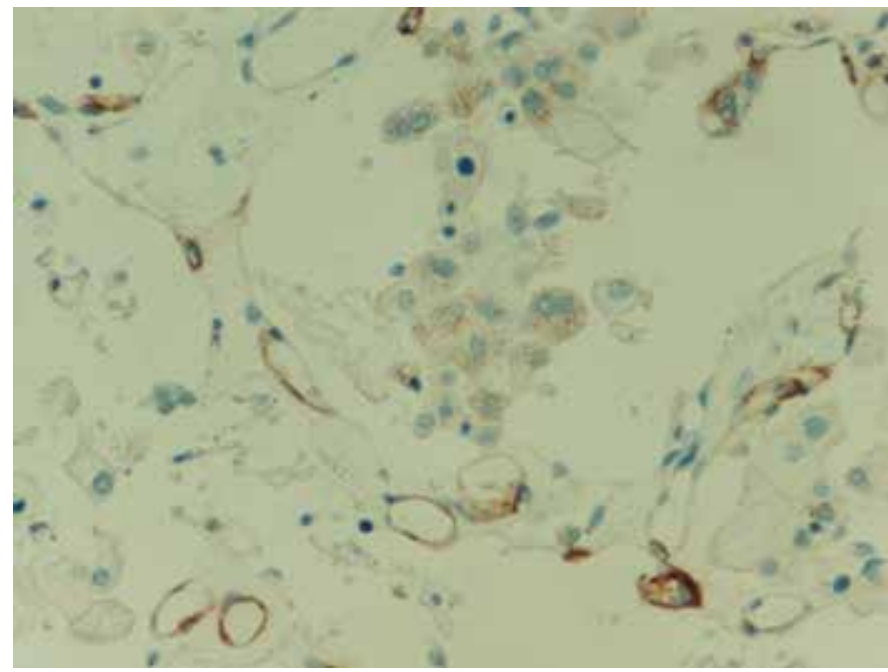

Image 6. Cytoplasmic granular collagen type XV alpha-1positive tumor cells (score 1) and microvascular structures in a chromophobe renal cell carcinoma patient (x400)

Table 1: Rates of cytoplasmic staining for Gremlin 1

\begin{tabular}{|l|l|l|l|l|}
\hline Diagnosis & Score 0 & Score 1 & Score 2 & Total \\
\hline PRCC & $16(80.0 \%)$ & $4(20.0 \%)$ & $0(0.0 \%)$ & $20(100 \%)$ \\
\hline CRCC & $8(20.5 \%)$ & $27(69.2 \%)$ & $4(10.3 \%)$ & $39(100 \%)$ \\
\hline
\end{tabular}

PRCC: Papillary renal cell carcinoma, CRCC: Chromophobe renal cell carcinoma

Table 2: Rates of cytoplasmic Gremlin 1 staining in normal kidney tissues of both tumor groups

\begin{tabular}{|l|l|l|l|l|}
\hline Diagnosis & Score 0 & Score 1 & Score 2 & Total \\
\hline Normal tubular epithelial tissue in PRCC patients & $7(35.0 \%)$ & $12(60.0 \%)$ & $1(5.0 \%)$ & $20(100 \%)$ \\
\hline Normal tubular epithelial tissue in CRCC & $3(7.7 \%)$ & $31(79.5 \%)$ & $5(12.8 \%)$ & $39(100 \%)$ \\
\hline
\end{tabular}

PRCC: Papillary renal cell carcinoma, CRCC: Chromophobe renal cell carcinoma 
COL15A1 expression did not differ statistically significantly with respect to necrosis in PRCC patients (necrosis present: 9 patients, necrosis absent: 11 patients). Tumor diameter did not show a statistically significant relationship with COL15A1 expression among CRCC patients.

Taking into account the fact that COL15A1 serves as an endothelial marker, the relationship between numerical values of microvascular structures (MVD) stained with COL15A1 and the pattern of cytoplasmic staining with GREM1 was compared between two tumor groups and found significant for PRCC $(\mathrm{p}=0.007)$ but non-significant for CRCC $(\mathrm{p}=0.147)$. Based on these results, a positive significant correlation was found between GREM1 and angiogenesis in PRCC but not in CRCC (Image 9).

Tumor necrosis was observed microscopically in $45 \%$ in PRCC patients and in 33\% of CRCC patients. Combined analysis of both tumor groups with respect to the tumor diameter and

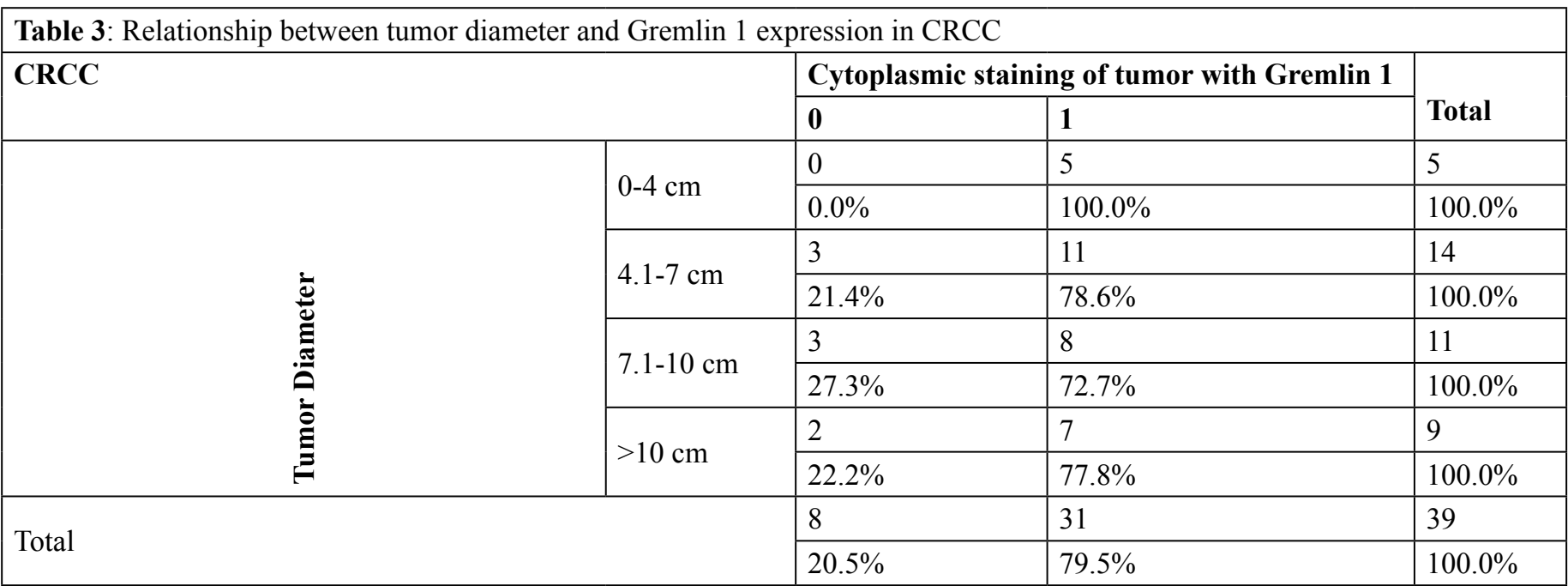

CRCC: Chromophobe renal cell carcinoma

Table 4: Cytoplasmic staining with COL15A in tumor tissues of both tumor groups

\begin{tabular}{|l|l|l|l|}
\hline Diagnosis & Score 0 & Score 1 & Total \\
\hline PRCC & $14(70 \%)$ & $6(30 \%)$ & $20(100 \%)$ \\
\hline CRCC & $38(97.4 \%)$ & $1(2.6 \%)$ & $39(100 \%)$ \\
\hline
\end{tabular}

PRCC: Papillary renal cell carcinoma, CRCC: Chromophobe renal cell carcinoma

Table 5: Relationship between tumor diameter and collagen type XV alpha-1 expression in papillary renal cell carcinoma

\begin{tabular}{|c|c|c|c|c|c|}
\hline \multicolumn{3}{|c|}{ PRCC } & \multicolumn{2}{|c|}{ COL15A1 tumor expression } & \multirow{3}{*}{$\begin{array}{l}\text { Total } \\
7\end{array}$} \\
\hline & & & Negative & Positive & \\
\hline & \multirow{8}{*}{ 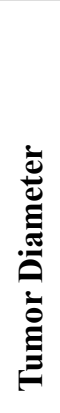 } & \multirow{2}{*}{$0-4 \mathrm{~cm}$} & 4 & 3 & \\
\hline & & & $57.1 \%$ & $42.9 \%$ & $100.0 \%$ \\
\hline & & \multirow{2}{*}{$4.1-7 \mathrm{~cm}$} & 5 & 2 & 7 \\
\hline & & & $71.4 \%$ & $28.6 \%$ & $100.0 \%$ \\
\hline & & \multirow{2}{*}{$7.1-10 \mathrm{~cm}$} & 3 & 0 & 3 \\
\hline & & & $100.0 \%$ & $0.0 \%$ & $100.0 \%$ \\
\hline & & \multirow{2}{*}{$>10 \mathrm{~cm}$} & 2 & 1 & 3 \\
\hline & & & $66.7 \%$ & $33.3 \%$ & $100.0 \%$ \\
\hline \multirow{2}{*}{\multicolumn{2}{|c|}{ Total }} & & 14 & 6 & 20 \\
\hline & & & $100.0 \%$ & $30,0 \%$ & $100.0 \%$ \\
\hline
\end{tabular}

PRCC: Papillary renal cell carcinoma, COL15A1: Collagen type XV alpha-1 
necrosis of the tumor tissue showed that necrosis was more likely to be found as tumor diameter increased and their association was statistically significant $(\mathrm{p}=0.035)$.

\section{Discussion}

Approximately 271,000 cases of kidney cancer were identified worldwide in 2008 with 116.000 people dying as a result of kidney cancer. RCC originating from renal parenchyma accounts for the majority of kidney cancers. Kidney cancers represent $2 \%$ of all adult malignancies. Among patients with kidney cancers, males are more commonly affected than females with a male to female ratio of 3:2 (8). In our study, the percentage of male patients was higher with a male to female ratio of 2.28.

The incidence of RCC peaks in the sixth decade of life and $80 \%$ of cases occur between the ages of 40 and 69 (9). Consistent with literature, the mean age of our patients was 54.9 years.

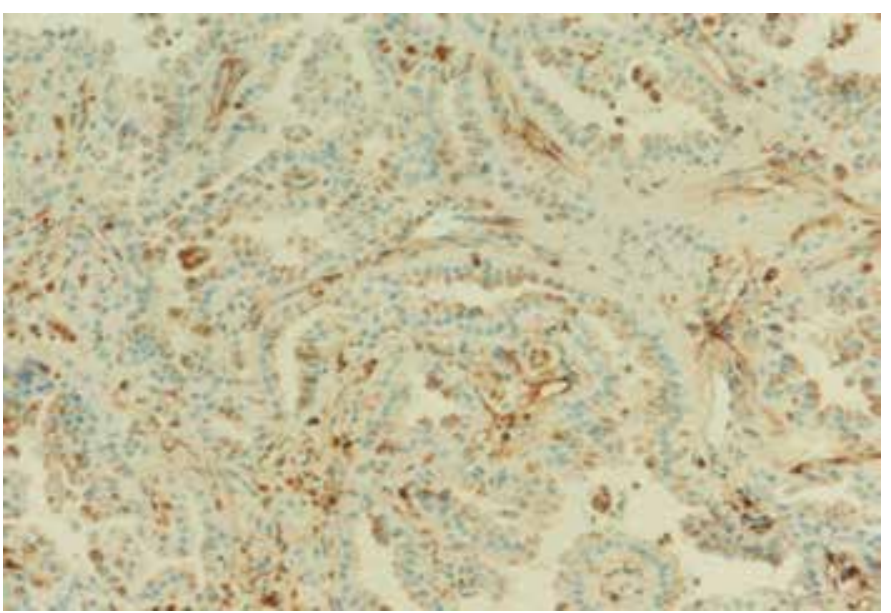

Image 7. Cytoplasmic granular collagen type XV alpha-1-positive tumor cells in a papillary renal cell carcinoma patient (x200)

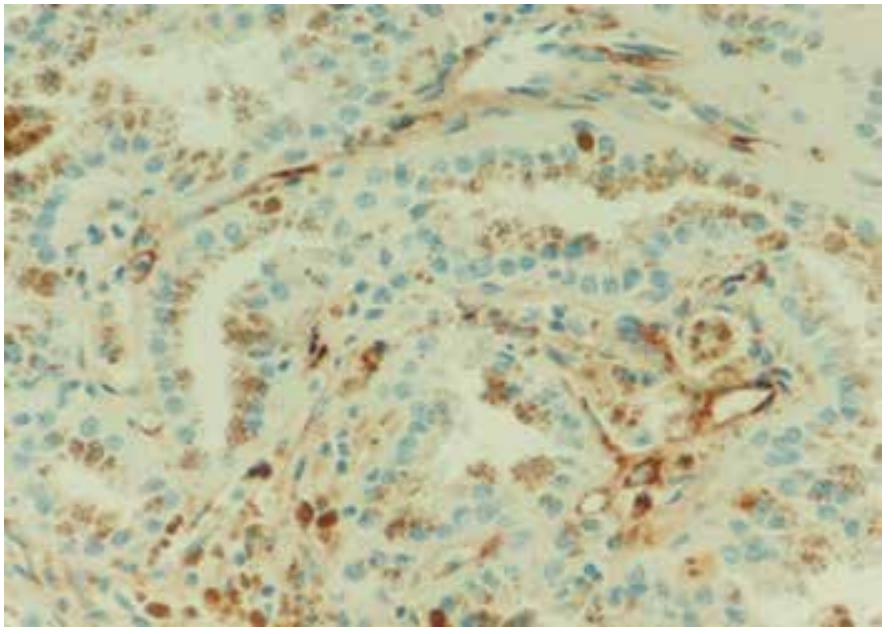

Image 8. Cytoplasmic granular collagen type XV alpha-1-positive tumor cells in a papillary renal cell carcinoma patient (score 2) $(\mathrm{x} 400)$
The mean tumor diameter is $6.4 \mathrm{~cm}$ in PRCC patients (10). In our study, the mean tumor diameter was $6.34 \mathrm{~cm}$ in PRCC patients, which was consistent with literature.

Intratumoral necrosis may be found in $32-75 \%$ of RCC patients (10). Tumor necrosis was observed microscopically in $45 \%$ of PRCC patients in our study.

The mean tumor diameter is $9 \mathrm{~cm}$ in CRCC patients (10). Our PRCC patients had a mean tumor diameter of $8.2 \mathrm{~cm}$. Tumor necrosis is found in one-third of CRCC patients (10). Tumor necrosis was observed microscopically in $33 \%$ of CRCC patients in our study.

Both genetic and epigenetic factors are known to play a role in renal carcinogenesis. Epigenetics are defined as changes in a gene's function that are inherited by meiotic or mitotic division without alterations in the DNA sequence (11).

Recent studies have demonstrated upregulation of GREM 1 in a variety of human cancers (12-15). In the present study, GREM1 expression did not differ statistically significantly between tumor tissue and neighboring intact tissue in PRCC patients but tumor tissue showed significantly higher GREM1 expression in CRCC patients ( $\mathrm{p}=0.006$ ). The percentage of tumor staining was $20 \%$ in PRCC patients versus $79.5 \%$ in CRCC patients. These findings suggest that GREM1 may have a prominent role as a tumor suppressor gene in the development of CRCC. Detailed genetic studies are needed to draw more definite conclusions on the putative role of GREM1 in CRCC tumorigenesis. We suggest that differential results obtained from the two tumor types examined in the present study might be explained by different genetic pathways involved in the development of these tumors.

In our study, COL15A1 expression was observed in the vascular structures and basement membranes of tumor tissues and

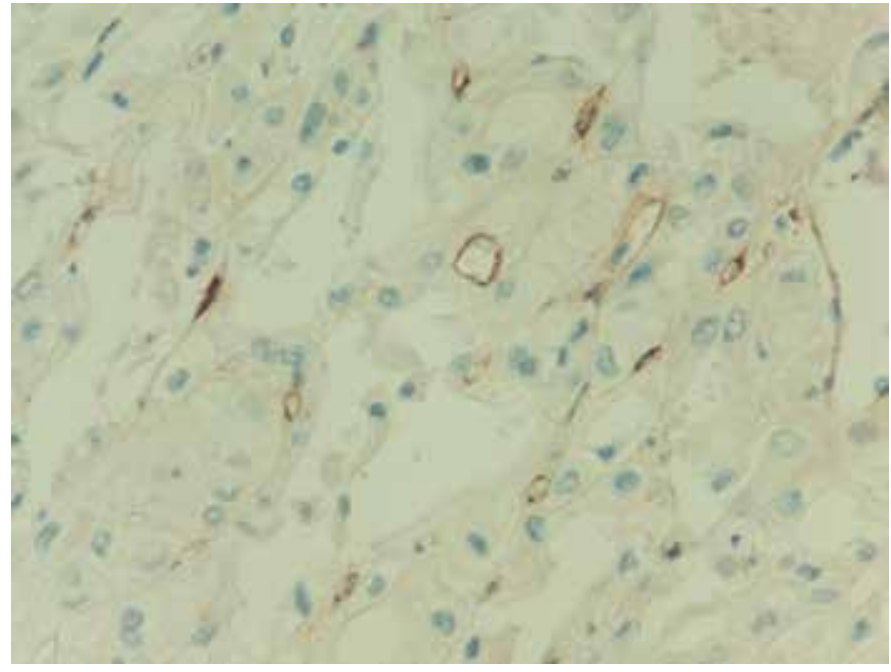

Image 9. Microvascular structures stained with collagen type XV alpha-1 in chromophobe renal cell carcinoma (x400) 
neighboring intact tissues in both tumor groups. Additionally, cytoplasmic staining of tumor zones was detected in 30\% of PRCC patients and $2.6 \%$ of CRCC patients. This percentage was statistically significant in PRCC $(p=0.0059)$. Since hypermethylation of the promoter region of the COL15A1 gene was previously reported in CCRCC patients $(4,5)$ concurrent genetic studies have to be conducted in order to determine whether cytoplasmic staining is related to hypermethylation. To our best knowledge, there are no immunohistochemical studies in literature that examined COL15A1 in any tumor tissue. Thus, our study is the first to report relevant findings on this issue.

GREM1 is a novel proangiogenic factor as demonstrated by several studies $(2,7)$. In a study by Chen et al. (7) on tumorrelated angiogenesis, expression of GREM1 was shown to be correlated with increased angiogenesis in patients with pancreatic neuroendocrine tumors. In a study by Chen et al. (7) it was argued that GREM1 might be used as a prognostic marker in pancreatic neuroendocrine tumors which was based on the positive correlation observed between increased MVD and GREM1 expression. On the other hand, Van Vlodrop et al. (1) reported that methylation of the promoter region of Gremlin was associated with higher tumor grade, higher tumor stage, reduced MVD and shorter survival time in patients with CCRCC. Karagiannis et al. (16) showed that GREM1 promotes the loss of cancer cell differentiation at the cancer invasion front, a mechanism that may facilitate tumor progression. Yan et al. (17) reported that Gremlin1-overexpressing cells display increased growth and tumor formation abilities. In our study, the association between GREM1 expression and angiogenesis was examined in tumor tissues using numerical values of microvascular structures (MVD) stained with COL15A1 taking into account the fact that COL15A1 serves as an endothelial marker. In PRCC patients, the number of microvascular structures showed a positive, significant association with GREM1 expression in the tumor tissue $(\mathrm{p}=0.007)$. Increased number of microvascular structures associated with GREM1 expression as observed in the current study suggested that GREM1 might be involved in increased angiogenesis in PRCC. For CRCC, a statistically significant association was not found between aforementioned parameters.

Increased angiogenesis is a characteristic feature of CCRCC. These tumors have the worst prognosis among all RCC cases. Given the fact that PRCC tumors are associated with a worse prognosis than CRCC tumors, it was suggested that a marker associated with increased angiogenesis in PRCC might serve as an indicator of poor prognosis. The association of GREM1 and COL15A1 markers with poor prognosis in RCC should be explored in future studies.

\section{Conclusion}

Cytoplasmic staining with GREM1 detected in both tumor groups, albeit at different rates, and much higher expression of this marker particularly in the tubular epithelium of the neighboring intact tissue support the hypothesis that GREM1 might be a tumor suppressor gene as suggested by literature data.

Significant relationship between the microvascular distribution pattern and GREM1 observed in PRCC patients suggest that GREM1 implicated in tumor angiogenesis might act as a proangiogenic factor in these tumors as well, in parallel with literature data.

We believe that the significant pattern of cytoplasmic staining in tumor tissues observed with COL15A1 particularly in PRCC cases in our study is of interest and this finding should be confirmed with additional studies.

It is our belief that genetic studies simultaneously conducted with immunohistochemical studies would provide more definite results on tumorigenesis in PRCC and CRCC and eventually contribute to the development of new diagnostic and therapeutic approaches by elucidating the underlying mechanism of these tumors.

\section{Acknowledgement}

We would like to thank to. Dr. Mithat Temizer from the Department of Public Health, Gaziantep University for her valuable contribution to statistical analyses. This study was funded by Gaziantep University Scientific Research Fund (Project No: FEF.11.10).

\section{Conflict of Interest}

The authors declare that there is no conflict of interest arising out of this manuscript.

\section{References}

1. van Vlodrop IJ, Baldewijns MM, Smits KM, Schouten LJ, van Neste L, van Criekinge W, et al. Prognostic significance of Gremlin1 (GREM1) promoter $\mathrm{CpG}$ island hypermethylation in clear cell renal cell carcinoma. Am J Pathol. 2010;176:575-584.

2. Stabile H, Mitola S, Moroni E, Belleri M, Nicoli S, Coltrini D, et al. Bone morphogenic protein antagonist Drm/gremlin is a novel proangiogenic factor. Blood. 2007;109:1834-1840.

3. Amenta PS, Scivoletti NA, Newman MD, Sciancalepore JP, Li D, Myers JC. Proteoglycan-collagen XV in human tissues is seen linking banded collagen fibers subjacent to the basement membrane. J Histochem Cytochem. 2005;53:165-176.

4. Morris MR, Ricketts C, Gentle D, Abdulrahman M, Clarke N, Brown $\mathrm{M}$, et al. Identification of candidate tumour suppressor genes frequently methylated in renal cell carcinoma. Oncogene. 2010;29:2104-2117. 
5. Yıldız K. Böbrek tümörlerinin patolojik sınıflamasında güncel gelişmeler. Üro-Onkoloji Bülteni. 2011;3:86-90.

6. Ellinger J, Holl D, Nuhn P, Kahl P, Haseke N, Staehler M, et al. DNA hypermethylation in papillary renal cell carcinoma. BJU Int. 2011;107:664-669.

7. Chen MH, Yeh YC, Shyr YM, Jan YH, Chao Y, Li CP, et al. Expression of gremlin 1 correlates with increased angiogenesis and progressionfree survival in patients with pancreatic neuroendocrine tumors. J Gastroenterol. 2013;48:101-108.

8. Ferlay J, Shin HR, Bray F, Forman D, Mathers C, Parkin DM. Estimates of worldwide burden of cancer in 2008: GLOBOCAN 2008. Int $\mathbf{J}$ Cancer. 2010;127:2893-2917.

9. Pascual D, Borque A. Epidemiology of kidney cancer. Adv Urol. 2008;2008:782381

10. Mills SE. Strenberg's Diagnostic Surgical Pathology. 5 ed. Wolters Kluwer, Lippincott Williams anda Wilkins; 2010;1757-1798.

11. Bora G, Yurter H. Epigenetic diseases and therapeutic approaches. Hacettepe Tip Dergisi. 2007;38:48-54.
12. Mulvihill MS, Kwon YW, Lee S, Fang LT, Choi H, Ray R, et al. Gremlin is overexpressed in lung adenocarcinoma and increases cell growth and proliferation in normal lung cells. PloS one. 2012;7:1-8.

13. Sneddon JB, Zhen HH, Montgomery K, van de Rijn M, Tward AD, West $\mathrm{R}$, et al. Bone morphogenetic protein antagonist gremlin 1 is widely expressed by cancer-associated stromal cells and can promote tumor cell proliferation. Proc Natl Acad Sci U S A. 2006;103:1484214847 .

14. Wang DJ, Zhi XY, Zhang SC, Jiang M, Liu P, Han XP, et al. The bone morphogenetic protein antagonist Gremlin is overexpressed in human malignant mesothelioma. Oncol Rep. 2012;27:58-64.

15. Namkoong H, Shin SM, Kim HK, Ha SA, Cho GW, Hur SY, et al. The bone morphogenetic protein antagonist gremlin 1 is overexpressed in human cancers and interacts with YWHAH protein. BMC Cancer. $2006 ; 6: 74$

16. Karagiannis GS, Musrap N, Saraon P, Treacy A, Schaeffer DF, Kirsch $\mathrm{R}$, et al. Bone morphogenetic protein antagonist gremlin-1 regulates colon cancer progression. Biol Chem. 2015;396:163-183.

17. Yan $\mathrm{K}, \mathrm{Wu} \mathrm{Q}$, Yan DH, Lee CH, Rahim N, Tritschler I, et al. Glioma cancer stem cells secrete Gremlin1 to promote their maintenance within the tumor hierarchy. Genes Dev. 2014;28:1085-1100. 